\title{
Verbesserte Labordiagnose der Herpes simplex Virus Infektion: Evaluierung eines modifizierten schnellen Shell-Vial-Assays zur Detektion und Typisierung von Herpes simplex Viren
}

\author{
Improved Laboratory Diagnosis of Herpes Simplex Virus Infection: Evaluation of a \\ Modified Rapid Shell-Vial-Assay for the Detection and Typing of Herpes Simplex Virus
}

\author{
H. F. Rabenau ${ }^{1,2}$, A. Helmerich ${ }^{1}$, B. Weber ${ }^{1,3}$
}

Zusammenfassung: Für eine rasche Labordiagnose der Herpes simplex Virus (HSV) Infektion ist ein schneller Erregernachweis von entscheidender Bedeutung. In der vorliegenden Arbeit wird eine schnelle, modifizierte Virusisolierung über den Nachweis von Virusstrukturproteinen mit Hilfe typenspezifischer monoklonaler Antikörper innerhalb von 36 Stunden (IPF-HSV) beschrieben. Es wurden insgesamt 560 Proben aus der Routinediagnostik vergleichend mit der konventionellen Virusisolierung über einen cytopathischen Effekt (CPE) und anschließender Typisierung (ZK-IFT) in infizierten Vero-Zellen und humanen Vorhautfibroblasten (HFF) untersucht. Die Sensitivität des IPF-HSV für HSV-1, bezogen auf den ZK-IFT (VeroZellen), betrug $96,8 \%$. Für HSV-2 wurde eine Sensitivität von $93,9 \%$ ermittelt. Die Spezifität des IPF-HSV lag für beide Virustypen über 99\% (HSV-1: 99,4\%, HSV-2: 99,1\%). Bezogen auf die konventionelle Virusisolierung im erweiterten Referenzstandard (Vero-Zellen und HFF) zeigte der IPF-HSV etwas geringere Werte für die Sensitivität (91,4\% für HSV-1; $91,6 \%$ für HSV-2). Unter den im Referenzstandard negativen Proben fanden sich mittels IPF-HSV eine HSV-1- und drei HSV-2-positive Proben. Die Spezifität lag für beide HSV-Typen über $99 \%$ (99,8\% für HSV-1, $99,4 \%$ für HSV-2). Bezogen auf den Referenzstandard ergab sich für den IPF-HSV ein positiv prädiktiver Wert von 97,0\% (HSV-1) bzw. 91,7\% (HSV-2); der negative prädiktive Wert des Tests lag jeweils bei $99,4 \%$. Die Ergebnisse unserer Studie zeigen, daß der IPF-HSV eine sinnvolle Alternative zur konventionellen Virusisolierung darstellt, da der Erregernachweis bereits nach 36 Stunden möglich ist. Der Testaufbau ist unter Verwendung geeigneter monoklonaler Antikörper und Zelltypen auch zur Schnelldiagnose respiratorischer Viruser-

\footnotetext{
${ }^{1}$ Institut für Medizinische Virologie der J.W.Goethe-Universität Frankfurt /Main

${ }^{2}$ Korrespondenzadresse: Priv. Doz. Dr. Holger F. Rabenau, Institut für Medizinische Virologie, Universitätskliniken Frankfurt, PaúlEhrlich Str. 40, D-60596 Frankfurt a. M. Fax: +49-69-6301-6477

${ }^{3}$ Laboratoires Réunis Kutter-Lieners-Hastert, Junglinster, Luxemburg

Eingegangen: 3. September 1999
}

krankungen, der Zytomegalie, dem kongenitalen Rötelnsyndrom und ggf. Rotavirusinfektionen geeignet.

Schlüsselwörter: Herpes simplex Virus/Isolierung und Anreicherung; Antigene, Virale/Immunologie; Immunenzymtechniken.

Summary: Rapid virus isolation is of considerable importance for laboratory diagnosis of herpes simplex virus (HSV) infection. In the present study, a modified rapid $(36 \mathrm{~h})$ virus isolation is described using the detection of structural proteins with type specific monoclonal antibodies (IPF-HSV). A total of 560 samples from the routine diagnostics were comparatively investigated with conventional virus isolation by cytopathogenic effect (CPE) and consecutive typing (ZK-IFT) in infected Vero-cells and human foreskin fibroblasts (HFF).The sensitivity of IPF-HSV for HSV-1 was $96.8 \%$ in comparison to ZK-IFT (Vero cells). The sensitivity for HSV2 was $93.9 \%$. The specificity for both virus types was higher than 99\% (HSV -1: 99.4\%, HSV-2: 99.1\%). IPFHSV showed slightly lower values for sensitivity (in comparison to the conventional virus isolation as expanded referenve standard (91.4\% for HSV-1; $91.6 \%$ for HSV-2). Among the samples that were negative in the reference standard, one and 3 , respectively, were tested positive for HSV-1 and HSV-2 -with IPF-HSV. The specificty was for both HSV types over $99 \%$ ( $99.8 \%$ for HSV-1, 99.4\% for HSV-2). The positive predictive values in comparison to the reference standard were $97.0 \%$ (HSV-1) and 91.7\% (HSV-2), respectively, the negative predictive value was $99.4 \%$ for both HSV types. The results of our study show that the IPF-HSV represents a valuable alternative to conventional virus isolation, since virus detection is already possible after $36 \mathrm{~h}$. By using appropriate monoclonal antibodies and cell types, the test principle is suited for the rapid diagnosis of respiratory virus diseases, cytomegalovirus infection, congenital rubella syndrome and rotavirus infections.

Keywords: Herpes Simplex Virus/isolation and purification; Antigens, Viral/immunology; Immunoenzyme Techniques. 
D ie Diagnose der Herpes simplex Virus (HSV) Infektion wird in den meisten Fällen über das charakteristische Erscheinungsbild der Effluoreszenzen gestellt. Verschiedene Erkrankungsbilder bedürfen aber einer weiteren Abklärung durch eine schnelle und zuverlässige Labordiagnose. Hierzu gehören lebensbe-drohliche Erkrankungen, wie die Herpes simplex Enzephalitis und der Herpes neonatorum. Des weiteren ist eine differentialdiagnostische Abgrenzung von Varizella zoster Virus (VZV) Infektionen bei immunsupprimierten Patienten von großer prognostischer und therapeutischer Bedeutung. Beim Herpes genitalis sollte zusätzlich zur Isolierung eine Typisierung durchgeführt werden, da HSV-2 im Gegensatz zu HSV-1 häufiger zu Rekurrenzen neigt und eine geringere Sensitivität gegenüber verschiedenen Nukleosidanaloga zeigt $[1,2]$. Seit vielen Jahren hat sich die konventionelle Virusisolierung über den Nachweis eines cytopathischen Effekts (CPE) mit anschließender Typisierung für die Labordiagnose der Herpes simplex Infektionen bewährt. In der Regel tritt ein CPE nach einer Inkubationszeit von 2-3 Tagen auf, allerdings ist bei hohem Infektionstiter eine Virusisolierung bereits nach 24-36 Stunden möglich. Wesentlich schneller als die konventionelle Virusisolierung gelingt der Erregernachweis über die Detektion von Virusstrukturproteinen im Patientenmaterial mit Hilfe der direkten Immunfluoreszenz (IFT) oder ELISA und über die Amplifizierung von Nukleinsäuresequenzen mit der Polymerasekettenreaktion (PCR). Der Antigennachweis mittels ELISA besitzt im Vergleich zur Virusisolierung und PCR eine geringere Sensitivität und ist nur eingeschränkt zur Typisierung geeignet. Die PCR ist das diagnostische Verfahren der Wahl zur Detektion geringster Mengen viraler DNA wie z. B. bei der Herpes Enzephalitis [3, 4]. Obwohl sie auch im Prinzip in puncto Sensitivität der Virusisolierung für den Erregernachweis bei Haut- und Schleimhautlokalisationen der HSV Infektion überlegen ist [5], steht ihrem Einsatz der zur Zeit noch relativ hohe Arbeits- und Kostenaufwand gegenüber.

In der vorliegenden Arbeit wird eine dem „shellvial"-Verfahren ähnliche Methode vorgestellt, welche die Dauer des Erregernachweises mit anschließender Typisierung im Vergleich zur herkömmlichen Zellkultur $(\mathrm{ZK})$ bei reduziertem Arbeitsaufwand eindeutig verkürzt. Aufbau und Durchführung wurden am Beispiel der Herpes-simplex-Viren (Typ 1 und 2) dargestellt. Die Sensitivität und Spezifität des Tests wurden mit dem konventionellen Anzuchtverfahren auf zwei verschiedenen Zellkulturen und anschließender Detektion mittels Immunfluoreszenztest verglichen. Der Aufbau der Testmethode stützt sich im wesentlichen auf frühere Arbeiten über die diagnostische Verwen-

Nicht standardisierte Abkürzungen: ATCC, American Type Culture Collection; CPE, cytopathischer Effekt; ELISA, enzyme-linked immunosorbent assay; HFF, human forskin fibroblast; HSV, Herpes simplex Virus; IFT, indirekter Immunfluoreszenztest; IPF, Immunoperoxydasefärbung; PCR, polymerase chain reaction; RSV, respiratory syncytial virus; VZV, Varizella Zoster Virus; ZK, Zellkultur. dung monoklonaler Antikörper [6-8]. Analog zur Shell-vial-Technik erfolgt eine Zentrifugation der Probe auf die Zellkultur; dies beschleunigt die Inokulation und verkürzt dadurch die Nachweiszeit [9] möglicherweise durch Einfluß auf die Bindung des Virus an die Zelloberfläche [10]. In der hier beschriebenen Versuchsreihe wurde die IPF-HSV-Methode exemplarisch an HSV-1 und -2 evaluiert.

\section{Methoden}

\section{Patientenproben}

Sämtliche untersuchten 560 Proben stammen aus dem Einsendegut des Institutes für Medizinische Virologie des Klinikums der J. W. Goethe-Universität, Frankfurt a. Main.

Unter den eingesandten Proben fanden sich $268 \mathrm{Ab}-$ striche von Effloreszenzen, 140 Rachenabstriche, 95 Liquorpunktate, 36 Bronchiallavagen, 15 Punktate aus Hautbläschen, 5 Urinproben und 1 Pleurapunktat. Urinproben wurden mit Hilfe einer Einwegspritze dem Einsendegefä $ß$ entnommen und unter $Z$ wischenschaltung eines Filters mit $0,45 \mu$ l Porengröße (Sterilfilter „Millex - HA“, Millipore, Eschborn) in sterile Aufbewahrungsgefäße abgefüllt. Bronchiallavagen sowie Sputum wurden zu gleichen Teilen mit Aufbereitungspuffer verdünnt. Alle Proben wurden nach Eintreffen im Labor aufbereitet und ohne zwischenzeitliches Einfrieren parallel auf herkömmliche Zellkulturen und Mikrotiterplatten aufgetragen.

Als Positiv-Kontrollen dienten HSV-Laborstämme (HSV-1: McIntyre, HSV-2: MS, American Type Culture Collection (ATCC), Rockville, Md., USA)

\section{Konventionelle Zellkultur und Virustypisierung}

Jedes Zellkultur-Flachboden-Röhrchen wurde mit $2 \mathrm{ml}$ Zellsuspension ( $2 \times 10^{4}$ Zellen/ml) beschickt und während 72 Stunden bei $37^{\circ} \mathrm{C}$ inkubiert. Anschließend erfolgte eine Beimpfung mit $200 \mu \mathrm{l}$ der vorbehandelten Probe. Die Röhrchen wurden mit 2 ml MEM (1\%) aufgefüllt und bei $37^{\circ} \mathrm{C}$ inkubiert. Jede Patientenprobe wurde sowohl auf humane Vorhautfibroblasten (HFF) als auch auf Affennierenzellen (Vero) verimpft.

Die beimpften Zellkulturröhrchen wurden täglich lichtmikroskopisch untersucht. Zeigte sich ein cytopathischer Effekt (CPE), wurde ein kommerziell verfügbarer, direkter Immunfluoreszenztest (IFT, Microtrak HSV 1/2 Typing Set, Syva/Dade Behring. Liederbach) zur Identifizierung und Typisierung von HSV entsprechend den Herstellerempfehlungen durchgeführt und interpretiert.

Immunperoxidasefärbung zur semiquantitativen Bestimmung von HSV-Strukturproteinen in infizierten Zellen (HSV-IPF)

In jede Vertiefung der Flachboden-Mikrotiterplatte (Greiner, Frickenhausen) wurden $100 \mu \mathrm{l}$ Vero-Zellsuspension $\left(2 \times 10^{4}\right.$ Zellen) pipettiert und während 72 Stunden bei $37^{\circ} \mathrm{C}$ in mit $5 \% \mathrm{CO}_{2}$ angereicherter At- 
mosphärc inkubiert. Für jede Probe wurden vier Vertiefungen mit jewcils 50 $\mu$ l Lysat beschickt, ohne das vorhandene Zellkulturmedium vorher zu entfernen.

Dic Platte wurde mit Abdeckfolic verschlossen und bei $700 \&$ während 30 min zentrifugiert. Dieser Schritt beschleunigt die Sedimentation der infektiösen Partikel auf den Zellrasen [11]. Anschließend wurden die beimpften Platten bei $37^{\circ} \mathrm{C}$ während $36 \mathrm{~h}$ inkubiert. Detektion und Typisicrung mit monoklonalen Antikörper wurden im Anschluß durchgeführt, olnne einen mikroskopisch sichtbaren CPE abzuwarten. Der Überstand wurde abgesaugt und durch je $100 \mu \mathrm{l}$ eines eisgekühlten Gemisches aus $40 \%$ Aceton und $60 \%$ Methanol ersetzt; nach 15 min Inkubation bei Raumtemperatur wurde die Flüssigkeit verworfen.

Je $50 \mu 1$ monoklonaler Antikörper gegen HSV-1 und HSV-2 spezifische Glykoproteine (Paesel \& Lorei, Frankfurt) wurden $1: 500$ verdünnt in Blocking Solution (Rinderserumalbumin, $20 \mathrm{~g} / \mathrm{l}$; Ziegenserum, 50 $\mathrm{ml} / \mathrm{l}$; Thimerosal, $20 \mathrm{ml}$ 1\%ige Lösg./l gelöst in Waschpuffer) im Doppelansatz hinzugegeben. Nach einstündiger Inkubation bei $37{ }^{\circ} \mathrm{C}$ wurden die Mikrotiterplatten dreimal mit Waschpuffer (Rinderserumalbumin, $1,0 \mathrm{~g} / \mathrm{l} ; \mathrm{NaCl}, 0,2 \mathrm{Mol} / \mathrm{l}$; Thimerosal, 0,1\%; Tris, $0.02 \mathrm{mmol} / 1$; Tween $20,0,2 \%$, i.e. $2 \mathrm{ml} / \mathrm{l}$ gelöst in Aqua bidest, $\mathrm{pH} 7,45)$ gewaschen. Anschließend wurden die im Verhältnis $1: 1000$ in Waschpuffer verdünnten Anti-Maus-IgG-Antikörper (50 $\mu \mathrm{l}$ je Loch) aufgetragen, $1 \mathrm{~h}$ bei $37^{\circ} \mathrm{C}$ inkubiert und erneut dreimal gewaschen. $50 \mu \mathrm{l} /$ Vertiefung vom StreptavidinPeroxidase-Konjugat, verdünnt 1:3.000 in Waschpuffer wurden hinzugegeben und $30 \mathrm{~min}$. bei $37^{\circ} \mathrm{C}$ inkubiert. Nach einem letzten Waschschritt wurde dem Substrat (100 $\mu \mathrm{l}$ AEC-Lösung bestehend aus Aminoethylcarbazol, $180 \mathrm{mg}$; N-N-Dimethylformamid, 45 $\mathrm{ml}$ und Acetatpuffer, $105 \mathrm{ml}) 1 \mu \mathrm{l} \mathrm{H}_{2} \mathrm{O}_{2} / \mathrm{ml}$ zugesetzt. Nach maximal 30 min bei $37^{\circ} \mathrm{C}$ ließen sich die infizierten Zellen lichtmikroskopisch erkennen; die typspezifischen monoklonalen Antikörper ermöglichten zusätzlich die Differenzierung zwischen HSV-1und HSV-2-Infektionen. Die typische rötliche Anfärbung bereits einer solitären Zelle wurde als positiver Virusnachweis bewertet.

\section{Auswertung}

In Vorversuchen hatte sich gezeigt, daß bei $14,3 \%$ der Proben eine Virusisolierung nur auf HFF gelang, während auf Vero-Zellen kein CPE zu beobachten war. Daher wurde der Parallelansatz beider Zellinien als „erweiterter Referenz-Standard“ zur Evaluierung von Sensitivität und Spezifität des IPF-HSV eingesetzt. Kulturen, welche nach 14 Tagen lichtmikroskopisch keinerlei CPE aufwiesen, wurden als „negativ“ bewertet.

Als „falsch-positiv“" wurden diejenigen Testergebnisse gewertet, die im IPF-HSV, aber nicht auf Vero-Zellen und nicht auf Vorhaut-Fibroblasten einen CPE zeigten. Wenn die Negativ-Kontrolle in einem der beiden Tests ebenfalls positiv reagierte, wurde von einer Kontamination ausgegangen und der Versuch wiederholt.
Als "falsch-negativ" wurden die Ansätze gewertet, die im IPF-HSV keine lichtmikroskopisch sichtbare Anfärbung, aber in mindestens einem der beiden Referenz-Ansätze (Vero-/ Vorhaut-Zellen) einen CPE mit IFT-Nachweis von HSV-I bzw. -2 aufwiesen.

\section{Ergebnisse}

\section{Konventionelle Virusisolierung}

Mit den herkömmlichen Zellkulturen (Referenz-Standard) ließ sich HSV aus 71 (12,7\%) Proben anzüchten. Im IFT fanden sich darunter zu fast gleichen Anteilen HSV-1 $(n=35)$ und HSV-2 $(n=36)$. Alle IFT-Ergebnisse waren zweifelsfrei auszuwerten und wiesen ein charakteristisches Fluoreszenzmuster auf. Wie erwartet, waren mit keiner der beiden Zell-Linien sämtliche Infektionen nachweisbar (Tabellen 1a-c).

Auf Vero-Zellen wurden 31 aller HSV-1- bzw. 33 aller HSV-2- positiven Proben isoliert $(88,6 \%$ bzw. 91,7\%). Vier HSV-1- und drei HSV-2-Infektionen konnten nur über die Beimpfung von HFF beobachtet werden (Tabellen la und $1 \mathrm{~b}$ ).

Auf HFF ließen sich 30 aller HSV-1- und 24 aller HSV-2-positiven Proben nachweisen $(85,7 \%$ bzw. $66,7 \%$ ). Im Gegensatz zum Isolierungsversuch auf Verozellen, wurden mit den HFF fünf HSV-1- und

Tabelle 1a Ergebnisse der konventionellen HSV-1 Isolierung und Typisierung: Verozellen und HFF im Vergleich

\begin{tabular}{lccc}
\hline & ZK-IFT HFF pos. & ZK-IFT HFF neg. \\
\hline ZK-IFT Vero positiv & 26 & 5 \\
ZK-IFT Vero negativ &. & 4 & 525
\end{tabular}

Tabelle 1b Ergebnisse der konventionellen HSV-2 Isolierung und Typisierung: Verozellen und HFF im Vergleich

ZK-IFTं HFF pos. ZK-IFT HFF neg.

\begin{tabular}{lrr}
\hline ZK-IFT Vero positiv & 21 & 12 \\
ZK-IFT Vero negativ & 3 & 524
\end{tabular}

Tabelle 1c Ergebnisse der konventionellen HSV-1 und $-2 \cdot$ Isolierung und Typisierung: Verozellen und HFF im Vergleich

\begin{tabular}{lcc}
\hline & $\begin{array}{c}\text { ZK-IFT HFF } \\
\text { positiv }\end{array}$ & $\begin{array}{c}\text { ZK-IFT HFF } \\
\text { negativ }\end{array}$ \\
\hline $\begin{array}{l}\text { ZK-IFT Vero positiv } \\
\text { ZK-IFT Vero negativ }\end{array}$ & 47 & 17 \\
$i$ & 7 & 489 \\
\hline
\end{tabular}


Tabelle 2a HSV-1. Vergleichende Gegenüberstellung der konventionellen Zellkultur (ZK-IFT) und IPF-HSV

\begin{tabular}{|c|c|c|c|c|}
\hline . & $\begin{array}{l}\text { ZK-IFT Vero positiv } \\
\text { ZK-IFT HFF positiv }\end{array}$ & $\begin{array}{l}\text { ZK-IFT Vero positiv } \\
\text { ZK-IFT HFF negativ }\end{array}$ & $\begin{array}{l}\text { ZK-IFT Vero negativ } \\
\text { ZK-IFT HFF positiv }\end{array}$ & $\begin{array}{l}\text { ZK-IFT Vero negativ } \\
\text { ZK-IFT HFF negativ }\end{array}$ \\
\hline IPF-HSV positiv & 26 & 4 & 2 & 1 \\
\hline IPF-HSV negativ & 0 & 1 & 2 & 524 \\
\hline
\end{tabular}

Tabelle 2b HSV-2. Vergleichende Gegenüberstellung der konventionellen Zellkultur (ZK-IFT) und IPF-HSV

\begin{tabular}{lcccc}
\hline & $\begin{array}{c}\text { ZK-IFT Vero positiv } \\
\text { ZK-IFT HFF positiv }\end{array}$ & $\begin{array}{c}\text { ZK-IFT Vero positiv } \\
\text { ZK-IFT HFF negativ }\end{array}$ & $\begin{array}{c}\text { ZK-IFT Vero negativ } \\
\text { ZK-IFT HFF.positiv }\end{array}$ & $\begin{array}{c}\text { ZK-IFT Vero negativ } \\
\text { ZK-IFT HFF negativ }\end{array}$ \\
\hline IPF-HSV positiv & 20 & 11 & 2 & 3 \\
IPF-HSV negativ & 1 & 1 & 1 & 521 \\
\hline
\end{tabular}

Tabelle 2c HSV-1 und -2: Vergleichende Gegenüberstellung der konventionellen Zellkultur (ZK-IFT) und IPF-HSV

\begin{tabular}{lcccc}
\hline & $\begin{array}{c}\text { ZK-IFT Vero positiv } \\
\text { ZK-IFT HFF positiv }\end{array}$ & $\begin{array}{c}\text { ZK-IFT Vero positiv } \\
\text { ZK-IFT HFF negativ }\end{array}$ & $\begin{array}{c}\text { ZK-IFT Vero negativ } \\
\text { ZK-IFT HFF positiv }\end{array}$ & $\begin{array}{r}\text { ZK-IFT Vero negativ } \\
\text { ZK-IFT HFF negativ }\end{array}$ \\
\hline IPF-HSV positiv & 46 & 15 & 4 & 4 \\
IPF-HSV negativ & 1 & 2 & 3 & 485 \\
\hline
\end{tabular}

zwölf HSV-2-Patientenstämme nicht isoliert (Tabellen la und $1 \mathrm{~b}$ ).

\section{Ergebnisse des IPF-HSV}

Der IPF-HSV wurde nur mit Vero-Zellen durchgeführt, da diese sich (erwartungsgemäß) in den Vorversuchen im Vergleich zu HFF als empfänglicher für eine HSV-Infektion gezeigt hatten. Im Gegensatz zu den Vorversuchen konnten nicht sämtliche der auf herkömmlichen Zellkulturen nachgewiesenen Infektionen mit dem IPF-HSV erkannt werden. Alle im IPF-HSV als "positiv" gewerteten Proben wiesen eine charakteristische Färbung mit monoklonalen Antikörpern auf; uneindeutige oder unspezifische Färbungen traten nicht auf. Vereinzelt wiesen die Zellen typische toxische Schäden auf (Lücken im Monolayer); die Ursache dürfte in den aufgetragenen Probenmaterialien liegen. In allen Fällen war jedoch mindestens $50 \%$ des Zellrasens intakt. Zum Referenztest diskrepante Ergebnisse traten bei keiner dieser Proben auf.

HSV-1 wurde mit der herkömmlichen Technik auf Vero-Zellen in 31 Fällen angezüchtet; eine dieser Proben reagierte negativ im IPF-HSV. Die Sensitivität des IPF-HSV für HSV-1, bezogen auf den ZK-IFT Vero, liegt demnach bei 96,8\%. In fünf Proben, die irn herkömmlichen Verfahren auf Vero-Zellen keinen CPE verursacht hatten, war mit dem IPF-HSV eindeutig HSV-1 nachweisbar (Tabellen 2a-c).

HSV-2 ließ sich mit der herkömmlichen Technik in 33 Fällen auf Vero-Zellen anzüchten; in zwei dieser
Proben ließ sich im IPF-HSV keine HSV-Infektion nachweisen. Dies entspricht einer Sensitivität von 93,9\%. Auch hier gelang in fünf Proben, die in der herkömmlichen Technik auf Vero-Zellen keinen CPE verursacht hatten, ein Nachweis von HSV-2 im IPFHSV $^{*}$ (Tabelle $2 b$ ).

Die Spezifität des IPF-HSV liegt für beide Virustypen über 99\% (HSV-1: 99,4\%, HSV-2 99,1\%).

Bezogen auf die konventionelle Virusisolierung im erweiterten Referenzstandard (Vero-Zellen und HFF) zeigt der IPF-HSV etwas geringere Werte für die Sensitivität (91,4\% für HSV-1; 91,6\% für HSV-2). Unter den im Referenzstandard negativen Proben fanden sich mittels IPF-HSV eine HSV-1- und drei HSV-2-positive Proben (Tabellen 3a-c). Diese Ergebnisse waren reproduzierbar.

Die Spezifität liegt für beide HSV-Typen über $99 \%$ (99,8\% für HSV-1, 99,4\% für HSV-2). Bezogen auf den Referenzstandard ergibt sich für den IPF-HSV ein positiv prädiktiver Wert von $97,0 \%$ (HSV-1) bzw. $91,7 \%$ (HSV-2); der negativ prädiktive Wert des Tests liegt jeweils bei $99,4 \%$.

\section{Ergebnisse ergänzender diagnostischer Tests und Spe- zifität des IPF-HSV}

Zwölf Proben des Test-Kollektivs verursachten in der herkömmlichen Zellkultur einen mikroskopisch sichtbaren CPE, der mittels IFT auf Adenoviren zurückgeführt werden konnte. Im IPF-HSV mit monoklonalen Antikörpern gegen HSV-1 bzw. -2 trat bei diesen Pro- 


\begin{tabular}{|c|c|c|}
\hline & IPF-HSV positiv & IPF-HSV negativ \\
\hline $\begin{array}{l}\text { ZK-IFT positiv } \\
\text { (Vero / HFF) }\end{array}$ & 32 & 3 \\
\hline $\begin{array}{l}\text { ZK-IFT negativ } \\
\text { (Vero / HFF) }\end{array}$ & 1 & 524 \\
\hline
\end{tabular}

\begin{tabular}{|c|c|c|}
\hline & IPF-HSV positiv & IPF-HSV negativ \\
\hline $\begin{array}{l}\text { ZK-IFT positiv } \\
\text { (Vero + HFF) }\end{array}$ & 33 & 3 \\
\hline $\begin{array}{l}\text { ZK-IFT negativ } \\
\text { (Vero + HFF) }\end{array}$ & 3 & 521 \\
\hline
\end{tabular}

Tabelle 3c Vergleich Referenzstandard/IPF-HSV für HSV-1 und -2 gesamt

\begin{tabular}{lcc}
\hline & IPF-HSV positiv & IPF-HSV negativ \\
\hline $\begin{array}{l}\text { ZK-IFT positiv } \\
\text { (Vero + HFF) }\end{array}$ & 65 & 6 \\
$\begin{array}{l}\text { ZK-IFT negativ } \\
\text { (Vero + HFF) }\end{array}$ & 4 & 485 \\
\hline
\end{tabular}

ben weder ein sichtbarer CPE noch eine Farbreaktion auf.

In zwei Proben ließ sich in der herkömmlichen Zellkultur VZV anzüchten. Eine Färbung im IPF-HSV bei Verwendung der monoklonalen Antikörper gegen HSV wurde bei beiden Proben nicht beobachtet.

Mittels Viusisolierung und Antigentests (ELISA) wurden außerdem alle Proben routinemäßig auf CMV, RSV (Respiratory Syncytial Virus), Influenzaviren sowie auf Chlamydien untersucht. CMV wurde in 5 Fällen nachgewiesen, RSV und Influenza (Typ B) in jeweils einem Fall und Chlamydien in zwei Fällen. Alle diese Proben zeigten ein eindeutig negatives Ergebnis im IPF-HSV sowohl für HSV-1 als auch für HSV-2.

Vergleich des Zeitaufwands zwischen konventionneller Zellkultur und IPF-HSV bis zum positiven Erregenachweis

Die Zellkulturen des Referenz-Standards wurden täglich auf mikroskopisch sichtbare Zeichen eines CPEs untersucht. Hinsichtlich der Dauer bis zum Auftreten von CPEs zeigte sich dabei unabhängig von der ZellLinie (Vero/HFF) eine deutliche Streuung.
Vergleicht man alle HSV-1-infizierten Proben, ergibt sich ein (arithmetischer) Mittelwert von 3,3 Tagen bis zum Eintreten eines CPE (Median 2 Tage, Streuung 1 bis 11 Tage, Standardabweichung 2.12). HSV-2infizierte Proben verursachten im Durchschnitt nach 3,7 Tagen einen CPE (Median 2 Tage, 1 bis 14 Tage, Standardabw. 2,78). Für beide Virustypen zusammen ergeben sich eine mittlere Inkubationszeit von 3,5 Tagen (Median 2 Tage; 1 bis 14 Tage, Standardabw. 2,51), (Abbildung 1).

Ermittelt man die Zeit bis zum Auftreten eines CPE separat für die 6 Proben, die im IPF-HSV falsch-negativ reagierten, finden sich vergleichsweise längere Zeitintervalle als bei den übereinstimmend positiven Proben. Die 3 im IPF-HSV falsch-negativen HSV-1Proben zeigten durchschnittlich nach 8,0 Tagen einen CPE im Referenz-Standard (min. 4, max. 11 Tage; Median 9 Tage), die im IPF-HSV positiven Proben bereits nach durchschnittlich 2,8 Tagen (min. 1, max. 6 Tage; Median 2 Tage).

Analog dazu wiesen die im IPF-HSV falsch-negativen HSV-2-Proben nach durchschnittlich 7,0 Tagen einen CPE auf (min. 5, max. 10 Tage; Median 6,0 Tage), die im IPF-HSV positiven Proben bereits nach durchschnittlich 3,4 Tagen (min. 1, max. 14 Tage; Median 2 Tage). Diese Unterschiede in den Anzuchtzeiten sind für HSV-1 und HSV-2 nicht statistisch signifikant ( $p=0,013$ bzw. $P=0,034$ ). Für beide Virustypen zusammen ergeben sich durchschnittlich 7,5 Tage (falsch negative) vs. 3,1 Tage (richtig positive; Median 7,5 vs. 2 Tage) mit signifikanter Differenz $\left(\mathrm{p}_{1+2}=0,0006\right)$.

Testergebnisse in Abhängigkeit von Probenmaterialien In 23 Abstrichen von peripheren Haut- oder Schleimhauteffloreszenzen, 7 Rachenabstrichen und 5 Bläschenpunktaten wurde HSV-1 isoliert. HSV-2 wurde in 32 peripheren Abstrichen, 2 Rachenabstrichen und 2 Bläschenpunktaten nachgewiesen. In keiner der eingesandten Bronchiallavagen, Urinproben, Liquorpunktate und Pleurapunktat waren mit ZK-IFT Vero, ZK-IFT

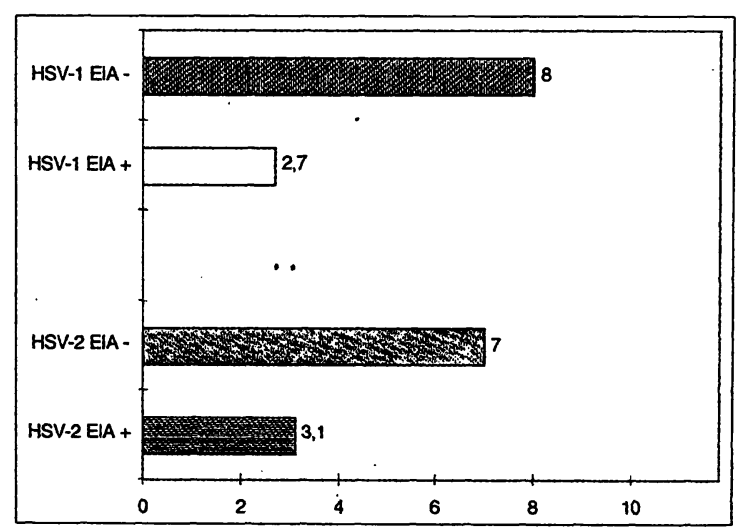

Abbildung 1 Inkubationszeiten bis zum Auftreten eines CPE durch HSV-1- bzw. HSV-2 im Referenztest (Vero oder HFF) in Abhängigkeit vom Ergebnis des IPF-HSV (EIA+: positives IPF-HSVErgebnis; EIA-: negatives IPF-HSV-Ergebnis). 
HFF und IPF-HSV vermehrungsfähige Herpes-simplex-Viren nachweisbar.

\section{Relation von klinischen Angaben zum Testergebnis} Unter den hier untersuchten 560 Proben waren insgesamt 468 (84\%) mit klinischen Angaben versehen, bei. 398 (71\%) war der Ort der Probenentnahme aus dem Anforderungsschein ersichtlich.

Eine Fragestellung, die ausdrücklich ,Herpes simplex“ benannte, lag bei 62 Proben vor (11\%), und zwar bei 23 (32\%) der 71 HSV-positiven Proben und bei 39 (8\%) der 489 HSV-negativen Proben. Die klassische Differentialdiagnose „VZV“ bzw. „Zoster" war bei 2 (3\%) der HSV-positiven Proben und 10 (2\%) der HSVnegativen Proben angegeben.

Eine HIV-Infektion als Grunderkrankung wurde bei 14 (20\%) der HSV-positiven Patientenproben genannt; darunter war bei 5 eine HSV-1-Infektion, bei 9 eine HSV-2-Infektion nachweisbar. Auch bei 24 (5\%) der HSV-negativen Proben lag eine HIV-Infektion vor.

20 (57\%) der HSV-1-Isolate stammten aus dem orolabialen und nasalen Bereich bzw. dem Rachen, 3 (9\%) aus dem genitoglutaealen Bereich, die übrigen von Effloreszenzen an Rumpf oder Extremitäten bzw. nicht näher lokalisierten Läsionen. Zwei Proben bestätigten ein Ekzema herpeticatum, in einem Fall ließ sich HSV-1 aus einem Panaritium eines HIV-infizierten Jungen isolieren.

24 (69\%) der HSV-2-Isolate stammten aus dem Genital- und Analbereich, 1 (3\%) aus dem Bereich der Nase, die übrigen von nicht näher bezeichneten Orten. In einem Fall konnte der klinische Verdacht auf einen M. Paget der Vulva als HSV-2-Infektion identifiziert werden.

\section{Diskussion}

Frühere Studien haben eindeutig die Vorteile der schnellen Virusisolierung mit Hilfe monoklonaler Antikörper genen Struktur- oder regulatorische Proteine verschiedener Viren gezeigt [7, 12].

Am Beispiel der Herpes-simplex-Viren Typ 1 und 2 konnte für die IPF-HSV-Testanordnung eine gute Sensitivität gezeigt werden. Bezüglich des Referenz-Standards (ZK-IFT Vero und HFF) findet sich ein gewisser Anteil falsch-negativer Ergebnisse (8,6\% für HSV-1; $8,3 \%$ für HSV-2).

Bei der Detektion von HSV-1 werden mit dem IPFHSV im gesamten Testkollektiv mehr positive Proben gefunden als mit dem ZK-IFT Vero bzw. dem ZK-IFT HFF (IPF-HSV: 33, ZK-IFT Vero: 31, ZK-IFT HFF: 30 Proben). Bei der Detektion von HSV-2 scheint der IPF-HSV ebenfalls etwas empfindlicher als der ZKIFT Vero, aber deutlich empfindlicher als der ZK-IFT auf HFF zu sein (IPF-HSV: 32, ZK-IFT Vero: 33, ZKIFT HFF: 24 Proben).

Ob der IPF-HSV tatsächlich eine höhere Sensitivität besitzt als die herkömmlich Zellkultur, läßt sich nur durch Prüfung gegen einen übergeordneten Teststandard, wie ihn beispielsweise die PCR darstellt, eva- luieren. Mehrere Arbeiten bestätigen Lücken der konventionellen Virusanzucht gegenüber molekularbiologischen Methoden [13, 14].

Unabhängig von der verwendeten Zellkultur- und Detektions-Methode sind falsch negative Ergebnisse auf eine unadäquate Probenentnahme und -transport zurückzuführen. Falsch-negative Ergebnisse kommen auch vor, wenn der Zeitpunkt der Probengewinnung zu spät (bzw. zu früh) gewählt wurde. So lassen sich bei Herpes-Effloreszenzen im Stadium der Verkrustung nur noch wenige oder keine infektiösen bzw. vermehrungsfähigen Partikel mehr nachweisen [15]. Falls klinisch oder epidemiologisch relevant, ist dann eine Suche nach Antigenstrukturen (Antigentest) oder Virus-DNA (mittels PCR) sinnvoll.

Bei immunkompetenten Personen ist eine Probe von einer Herpes-simplex-verdächtigen Läsion innerhalb von drei Tagen nach deren Auftreten zu gewinnen, im Falle einer Immunsuppression oder -defizienz des Patienten kann diese Zeitspanne verlängert sein. Auch bei anderen Virus-Infekten ist die Zellkultur bei Probenabnahme in der Rekonvaleszenz meist nicht mehr aussagekräftig.

Auch die Art und Qualität der verwendeten Zellen sind für das Testergebnis von entscheidender Wichtigkeit; so beeinflussen zunehmendes Alter und höhere Passagezahl der Zellkulturen die Suszeptibilität für Viren negativ [16].

Weitere Fehlerquellen stellen die jeweils verwendeten Antikörper in IFT und IPF-HSV dar. Die von den Immunglobulinen erfaßten Antigene sind in der Regel bei einem Großteil der Virus-Wildstämme präsent. Der Nachweis bestimmter Mutanten mit veränderter Glykoproteinstruktur kann jedoch bei Verwendung monoklonaler Antikörper fehlschlagen [17, 18].

Die Wahrscheinlichkeit, aus einer Probensuspension ein oder mehrere infektiöse Partikel zu isolieren, folgt einer Binomial-Verteilung. Demnach ist es bei einem niedrigen Virustiter im Probenmaterial von großer $\mathrm{Be}$ deutung, ob $200 \mu$ l (herkömmliche Zellkultur) oder 50 $\mu l$ (IPF-HSV) in den Test eingesetzt werden. Bei 25 Viren in $2 \mathrm{ml}$ Suspension liegt die Wahrscheinlichkeit, mindestens ein Virus in das Testgefäß zu übertragen, im IPF-HSV $(50 \mu \mathrm{l})$ unter $50 \%$, in der herkömmlichen Zellkultur $(200 \mu \mathrm{l})$ bereits über $90 \%$. Dieser Nachteil wurde teilweise ausgeglichen durch Verwendung eines Doppelansatzes im IPF-HSV, so daß je $100 \mu$ l Probensuspension übertragen wurden. (Nach Abschluß dieser Arbeit wurde auf einen 4-fach Ansatz übergegangen, so daß der Nachteil der geringeren Probenmenge nicht mehr zum Tragen kommt).

In den Referenz-Zellkulturen war die Zeit bis zum Auftreten eines CPE signifikant länger bei den Proben, die im IPF-HSV ein falsch-negatives Ergebnis zeigten. Insgesamt handelt es sich hierbei nur um wenige Proben. Dennoch läßt sich anhand der Daten vermuten, daß Material mit niederigem Infektionstiter im IPF-HSV falsch negativ wird: Andererseits wurde mit dem IPFHSV in einem Fall HSV-2 in Abstrichmaterial gefunden, welches in der herkömmlichen Zellkultur erst nach 14 Tagen einen eindeutigen CPE hervorgerufen hatte. 
Bezüglich des Zeit- bzw. Arbeitsaufwandes zeigt der ZK-IFT gegenüher der herkömmlichen Zellkultur-Methodik gewisse Vorteile. Aufgrund der gelegentlich iiber eine Woche betragenden Zeitspanne bis zum Auftreten eines CPE müssen herkömmliche Zellkulturen bis zu zwei Wochen inkubiert werden. Nach einer Woche ist eine Blindpassage der inkubierten Zellen durchzuführen. Dic Inkubation aller Proben eines IPFHSV-Ansatzes wird dagegen nach einer definierten Zeit (im vorliegenden Fall $36 \mathrm{~h}$ ) unterbrochen, wodurch eine parallele Bearbeitung höherer Zahlen von Proben ermöglicht wird. Dieser Vorteil wird teilweise dadurch relativiert, daß sämtliche Proben das Detektionsverfahren mit monoklonalen Antikörpern durchlaufen müssen. IIn Gegensatz zum IFT ist dabei eine größere Zahl von Arbeitsschritten notwendig. Auf die mikroskopische Auswertung kann weder beim ZK-IFT noch beim IPF-HSV verzichtet werden. Die Betrachtung des gesamten Zellrasens in einem FlachbodenRöhrchen beansprucht die doppelte bis dreifache Zeit (1-2 Minuten) im Vergleich zur Betrachtung einer Kultur im Mikrotiter-Format (max. 30 Sekunden) und muß prinzipiell täglich durchgeführt werden. Hinzu kommt die IFT-Auswertung positiver Proben im Dunkelfeld.

Deutlich schnellere Testergebnisse als mit dem IPFHSV lassen sich nur mittels Antigennachweis ohne vorherige Anzucht erreichen, wodurch jedoch Schwächen in Sensitivität und Spezifität in Kauf genommen werden $[19,20]$.

Bezüglich der Lokalisation der einzelnen Serotypen bzw. Spezies wurde das "klassische“ Verteilungsmuster bestätigt, soweit der Ort der Probenentnahme vermerkt war: Die Mehrzahl der HSV-1-Infektionen wurde im orolabialen Bereich nachgewiesen, die Mehrzahl der HSV-2-Infektionen im genitoglutealen Bereich.

Der hier vorgestellte Test läßt sich mittels entsprechender Auswahl von Antikörpern auf eine große Zahl in vitro kultivierbarer Viren anwenden. Voraussetzung ist die Verwendung entsprechend sensibler Zellen. Die hier verwendeten Vero-Zellen kommen in konventionellen Zellkulturen neben HSV auch für die Diagnostik von Röteln (Rubella), und Poliovirus sowie, bei geringerer Empfänglichkeit, Masern-, Reo-, Coxsackie $\mathrm{B}$ - und ECHO-Viren zum Einsatz.

Da im Gegensatz zur herkömmlichen Zellkultur, im IPF-HSV alle Proben dem gleichen Verfahren unterliegen, ist prinzipiell eine vollautomatische Durchführung möglich. Diesem Aspekt kommt auch das gewählte Mikrotiterplatten-Format entgegen. Eine Färbereaktion des freien Substrates mit kolorimetrischer, quantitativer Auswertung, wie sie in experimentellen Arbeiten beschrieben ist [21], kann die mikroskopische Auswertung ersetzen. Um im Falle einer einzelnen infizierten Zelle eine ausreichende Färbung zu erhalten, muß eine entsprechende Verstärkung des Signals gesichert sein.

\section{Danksagung}

Für hervorragende technische Hilfe danken wir Frau Gaby Bauer.

\section{Literatur}

1. Arvin AM, Prober CG. Herpes simplex virus. In: Murray PR. Manual of Clinical Microbiology $7^{\text {th }}$ ed. Washington DC: ASM Press, 1999, 878-87.

2. Rabenau HF, Weber B, Doerr HW. Epidemiology of herpes simplex virus type 2 (Herpes genitalis). Intervirology (im Druck). 3. Kessler HH, Pierer K. Weber B. Sakrauski A. Santner B. Stuenzner D, Gergely E, Marth E. Detection of herpes simplex virus DNA from cerebrospinal fluid with polymerase chain reaction and a rapid, nonradioactive hybridization technique. J Clin Microbiol 1994;32:1881-6.

4. Sakrauski A, Weber B, Kessler HH, Pierer K, Doerr HW. Comparison of two hybridisation assays for the rapid detection of PCR amplified HSV genome sequences from cerebrospinal fluid. J Virol Meth 1994;50:175-84.

5. Waldhuber MG, Denham I. Wadey C, Leong-Shaw W, Cross GF. Detection of herpes simplex virus in genital specimens by type-specific polymerase chain reaction. Int J STD AIDS 1999;10: 89-92.

6. Goldstein LC, McDougall J, Hackmann R.. Monoclonal Antibodies to Cytomegalovirus: Rapid Identification of Clinical Isolates and Preliminary Use in Diagnosis of Cytomegalovirus Pneumonia. Infection and Immunity 1982;38: 273-81.

7. Schacherer C, Braun W, Bauer G, Doerr HW. Detection of Cytomegalovirus in Bronchial Lavage and Urine Using a Monoclonal Antibody to an HCMV Early Nuclear Protein. Infection 1988;5:288-92.

8. Swenson PD, Kaplan MH. Rapid Detection of Cytomegalovirus in Cell Culture by Indirect Immunoperoxidase Staining with Monoclonal Antibody to an Early Nuclear Antigen. J Clin Microbiol 1985;21: 669-73.

9. Osborn JE, Walker DL. Enhancement of infectivity of murine cytomegalovirus in vitro by centrifugal inoculation. J Virol 1968; 2: 853-8.

10. Hodkgin PD, Scalzo AA, Swaminathan N, Price P, Shellam GR. Murine cytomegalovirus binds reversibly to mouse embryo fibroblasts: implications for quantitation and explanation of centrifugal enhancement. J Virol Meth 1988;16: 298-310.

11. Thiele MG, Bicak MS, Young A, Kinsey J, White RJ, Purtilo DT. Rapid detection of Cytomegalovirus, centrifugation and immunofluorescence with a monoclonal antibody to an early nuclear antigen. J Virol Meth 1987; 16: 327-38.

12. Weber B, Harms F, Selb B, Doerr HW. Improvement of rotavirus isolation in the cell culture by immune peroxidase staining. $J$ Virol Methods 1992;38:187-94.

13. Cone RW, Hobson AC, Brown Z, Ashley R, Berry S, Winter C, Corey $\mathrm{L}$. Frequent detection of genital herpes simplex virus DNA by polymerase chain reaction among pregnant women. JAMA 1994;272:792-6.

14. Thomas CA, Smith SE, Morgan TM, White WL, Feldman SR. Clinical application of polymerase chain reaction amplification to diagnosis of herpes virus infection. Am. J. Dermatopathol. 1994;16:268-74.

15. Döller G. Virusanzucht, Schnellidentifizierung und Direktnachweis viraler Antigene. Mikrobiologe 1996;6:3-7.

16. Lennette EH, Schmidt NI,., editors.. Diagnostic procedures for viral, rickettsial and chlamydial infections ( $5^{\text {th }}$ ed.). Washington: American Public Health Association, 1979.

17. Pererira L, Dondero DV, Gallo D, Devlin V, Woodie JD. Serological analysis of herpes simplex viruses 1 and 2 with monoclonal antibodies. Infect Immun 1982;35: 363-7.

18. Webster, RG, Kendal AP, Gerhard W. Analysis of antigenic drift in recently isolated influenza A (HIN1) viruses using monoclonal antibody preparations. Virology 1985;143:583-94.

19. Brinker JP, Herrmann JE. Comparison of three monoclonal antibody-based enzyme immunoassays for detection of herpes simplex virus in clinical specimens. Eur J Clin Microbiol Infect Dis 1995;14:314-7.

20. Goodyear HM, Wilson P, Cropper L, Laidler PW, Sharp IR, Thaker U, Kenny PA, Price EH, Harper JI. Rapid diagnosis of cutaneous herpes simplex infections using specific monoclonal antibodies. Clin Exp Dermatol 1994;19: 294-7.

21. Langlois $M$, Allard JP, Nugier F, Aymard M. A rapid and automated colorimetric assay for evaluating the sensitivity of herpes simplex strains to antiviral drugs. J Biol Standardization 1986: 14:201-11. 